\title{
Scheme Design of Railway Predictive Maintenance Based on IOT and AI Technology
}

\author{
Cheng Zhang ${ }^{1, *}$, Xiang Xie ${ }^{2,}$ and Xiaochun Guo ${ }^{3}$ \\ ${ }^{1}$ School of Economics and Management, Beijing Jiaotong University, Weihai Campus, Beijing, China \\ ${ }^{2}$ School of Economics and Management, Beijing Jiaotong University, Beijing, China \\ ${ }^{3}$ Shenyang Open University, Shenyang, China \\ *Corresponding author. Email: 20711033@bjtu.edu.cn
}

\begin{abstract}
With the rapid development of railway, the predictive maintenance of railway is more and more worthy of study. This paper analyses the development process of railway maintenance in China, evaluates the traditional application of corrective maintenance, analyses the mature application of preventive maintenance, and expounds that predictive maintenance has been applied. This paper analyses the benefits from four aspects: saving cost, reducing unexpected downtime, prolonging asset life cycle and enhancing customer satisfaction, and summarizes the key success factors of predictive maintenance from the aspects of method, scope, team and change, especially designs predictive maintenance priority matrix for selection of maintenance objectives. Finally, the paper summarizes and designs the architecture of predictive maintenance platform based on Internet of things and AI. This paper will provide a meaningful scheme reference for China Railway to formulate predictive maintenance strategy and vigorously promote this work.
\end{abstract}

Keywords: Predictive maintenance, Railway, IOT, Big data, AI

\section{INTRODUCTION}

According to the outline of 'the advanced railway planning for a powerful transportation country in the new era', which released in 2021, by 2035 , the railway network in China will reach about 200,000 km, including about 70,000 km of high-speed railway. Cities with a population of more than 200,000 realize railway coverage, of which cities with a population of more than 500,000 are accessible by high-speed railway. The national 1, 2 and 3-hour high-speed rail travel circle and the national 1,2 and 3-day fast cargo logistics circle have been fully formed ${ }^{[1]}$. It is premise to strive to achieve $100 \%$ safety guarantee to meet the requirements of rapid development of China's railway. How to do a good job in railway safety maintenance is an important research topic.

It is essential to carry out efficient maintenance strategy in order to improve service quality and reduce the cost of operations. This has governed the evolution of maintenance strategies of Railway assets globally. Such a strategy can ensure that there is no asset breakdown and disruption of operations. If planned well, maintenance can increase the availability, reliability and effectiveness of the asset.

Over the years, business enterprises have relied upon various maintenance techniques to manage the lifecycle of their physical assets, including Breakdown Maintenance, Preventive Maintenance, Total Productive Maintenance, Run-to-Failure Maintenance, ReliabilityCentred Maintenance, Predictive Maintenance, among others.

\section{AS-IS ANALYSIS OF RAILWAY ASSETS MAINTENANCE}

Total railway investment remains stable in China, the change of investment structure is taking place, and the growth space of equipment and maintenance industry is very broad. For example, depending on different models, the four-level maintenance cycle is about 3-6 years and the five-level maintenance cycle is about 6-12 years. With the vehicles gradually entering the four or five level overhaul cycle, the maintenance business of EMU will continue to grow in demand. 
With the rapid growth of railway maintenance, railway maintenance technology also needs to be constantly updated. Railways traditionally rely on preventive maintenance, it is now gradually transitioning to predictive maintenance.

\subsection{Corrective Maintenance is a Traditional Railway Maintenance Technology}

Corrective maintenance is a reactive maintenance technology that takes action after an asset failure occurs. Corrective maintenance is mainly used to supplement other maintenance technologies. This is because independent predictive maintenance is expensive because it requires maintaining a large inventory of spare parts. This increases inventory and labour costs, resulting in suboptimal asset productivity. However, it is difficult to get a grip on Predictive Maintenance without understanding the different maintenance techniques included corrective maintenance that the Railways have adopted on the way to its transition to predictive maintenance

In addition, due to its reactivity, corrective maintenance means interruption during normal operation. For example, if corrective maintenance is carried out on the track, because the track is maintained / fixed only after derailment, it will completely confuse the train operation and timetable. What is worse, if a passenger train accident occurs, it may also cause loss of life, and if a freight train accident occurs, it may also cause loss of freight owners. Therefore, it's essential to adopt preventive maintenance than corrective maintenance technology.

\subsection{Planned Preventive Maintenance is Applied Maturely}

In Preventive Maintenance, repairs or replacements are prescheduled on the basis of either the time or the amount of use the asset has been under operation. This kind of planned maintenance has been widely applied.

In time-based scheduling, the time interval is calculated according to historical data (such as the average life of assets), while in use based scheduling, the time interval is calculated according to the service time of assets (such as the mileage of trains). Compared with time-based scheduling, usage based scheduling is a better measurement form, because it ignores the time when the asset is in the ideal state and only considers the time when the asset is actually used. ${ }^{[2]}$ Therefore, using preventive maintenance, the time interval between train bearing lubrication can be determined according to the distance travelled by the train.

Preventive maintenance is significantly better than corrective maintenance because assets are maintained before failure, reducing downtime and improving availability. For the same reason, it helps to avoid the possible loss of life caused by passenger trains and the possible commercial loss caused by freight trains to freight owners.

However, planned preventive maintenance is not cost-effective. This is because it will be replaced and repaired whether it is necessary or not. Maintenance decisions are mainly based on the planned schedule rather than the actual condition of the asset, ignoring other conditions such as the operating environment and use purpose, which will also affect the work of the asset. For example, freight trains work differently from passenger trains. Therefore, the two types will have different maintenance requirements. The preventive maintenance technology may not take into account the differences in wheel maintenance requirements of passenger and freight trains. In order to overcome these shortcomings, maintenance technology should rely less on historical data and more on the actual condition of assets.

\subsection{Predictive Maintenance is Developing}

In maintenance practice, it is particularly necessary to make maintenance decisions according to the actual use and operation of assets. At ordinary times, assets are monitored regularly, and they only need to be replaced when the asset status needs to be changed. Compared with preventive maintenance, condition based maintenance reduces the frequency of maintenance because it is not based on plan, but depends on asset status. In addition, big data analysis is introduced into predictive maintenance decision-making, which improves the accuracy of technology and makes it more reliable.

As a relatively new practice, predictive Maintenance has made inroads in several developed countries and is now being adopted in the developing countries. Predictive Maintenance is now being used to make railway maintenance operationally efficient and costeffective.

With the rapid development of Railways, there are many challenges such as the pressure of increasing maintenance costs, narrow maintenance windows, nonavailability of talent for remote condition monitoring, and heightened customer expectations, it is the best possible solutions for Predictive Maintenance, which would enhance utilization, reduce costs, and safeguard the durability of railway networks.

\section{BENEFIT ANALYSIS OF PREDICTIVE MAINTENANCE}

Predictive Maintenance uses a combination of realtime asset monitoring and data analysis for taking maintenance decisions. It involves deployment of 
sensor-based diagnostic monitoring in real time for assets and sub-systems that provide enough time to act based on the recorded condition parameters. Sensors are placed along railway tracks and mounted on rolling stock to remotely capture critical parameters associated with rolling stock and fixed infrastructure respectively on a real-time basis. Along with the asset-related data, other data related to weather and geographical conditions is also captured for superior asset management.

The data captured across the network of sensors is fed into a predictive analysis model to draw conclusions about when a failure is likely to happen, the possible nature of the failure, and the consequent right time for undertaking maintenance. This enables proactive maintenance operations, thereby eliminating unplanned downtime of assets. Additionally, accurate forecasting of spare parts requirement through predictive analytics helps optimise procurement and shipping costs. Accurate insights on manpower requirements also help drive precise maintenance crew scheduling, helping the Railways become a lean mean machine.

Predictive Maintenance technique predicts the maintenance requirement for an asset before the default occurs, thereby providing benefits such as reduced cost, increased asset productivity, and increased lifetime of assets, among others. Some of the major benefits of Predictive Maintenance include:

\subsection{Saving Cost}

Sudden failure of railway equipment will lead to a series of serious problems, including damage of raw materials, scrapping of parts of machinery and equipment, maintenance cost of machinery and equipment and so on. By adopting the predictive maintenance solution of machinery and equipment, it can effectively reduce the sudden failure rate of machinery and equipment, promote the enterprise from expensive maintenance to economic predictive maintenance, and save time, manpower and other resource costs for the enterprise.

\subsection{Reduce Unexpected Downtime}

Predictive Maintenance helps avert any unexpected failure, reducing downtime of assets, in fact effectively increasing their runtime. For example, the sensors installed on the railway equipment detect various physical information, such as current intensity, vibration, speed, temperature, humidity level, etc. through data collection, prediction and analysis, the possible faults of the equipment and the causes of the faults can be known in advance, and the warning can be given in advance when the service life of the parts is approaching, This allows engineers to repair when the machine is offline or dormant, reduce downtime and improve productivity: reduce unexpected downtime by identifying problems before they cause the whole system to fail. Reducing scheduled manual inspections can also improve operational efficiency. ${ }^{[3]}$

\subsection{Extended Lifetime of Assets}

Predictive analysis enables manufacturers to perform root cause analysis and find the problem before the machine or asset stops running. Predictive Maintenance increases the life span of the assets and reduces the need for repairs and replacement by bringing in efficiency to the technique. ${ }^{[4]}$ This is particularly important for the Railways which is critically dependent on its assets. Predictive maintenance ensures good health of these assets and keeps them functioning for as long as possible, increasing its return on investment.

\subsection{Enhance Customer Satisfaction}

There are various competing modes of transport, it is important to enhance customer satisfaction for railways. Customer loyalty will depend upon a quality and reliable customer experience. ${ }^{[5]}$ For instance, passengers will use rail service more if their experience is satisfactory most of the time, there are no delays or breakdowns, and a robust infrastructure promises total safety.

\section{KEY SUCCESS FACTORS OF RAILWAY PREDICTIVE MAINTENANCE STRATEGY}

Based on the experience of adopting predictive maintenance technology in railway departments all the world, there are four key elements to develop a successful predictive maintenance strategy.

\subsection{Select Reasonable Promotion Methods}

From the perspective of data flow for developing predictive maintenance solutions, it can be divided into "top-down" and "bottom-up" methods.

The "top-down" approach refers to clearly defining business objectives firstly, and then determining and preparing the required data sets. The data collection process aims to develop predictive maintenance solutions, and the captured data should be consistent with the requirements of the solutions. The data sets required by this method are relatively clear and easy to understand. In the early development stage of predictive maintenance, it is a more appropriate and effective application method.

The "bottom-up" approach refers to a series of data mining explorations based on a large amount of data currently available to obtain feasible insights and patterns. This method has high requirements for data 
acquisition, processing and algorithm ability. It needs all kinds of basic capabilities to accumulate to a certain level, then its application effect could be fully guaranteed.

\subsection{Define Predictive Maintenance Requirements Clearly}

During the process of building a predictive maintenance solution, it is the most critical step to define the specific requirements covering the scope of the predictive maintenance solution. It is better to determine more accurately what is predictable rather than trying to predict everything.

The goal of predictive maintenance is all the failures of the most critical assets or systems, but sometimes it may not be possible to collect and analyse the data of each component, because some components may produce too much data, while others may not produce enough data for analysis. Therefore, the key components to be analysed are determined based on two main factors. First, the significance of components in asset functions, and second, their ability to generate data. Based on these two factors, a predictive maintenance priority matrix is established for use for considering predictive maintenance strategy, as shown in Figure 1.

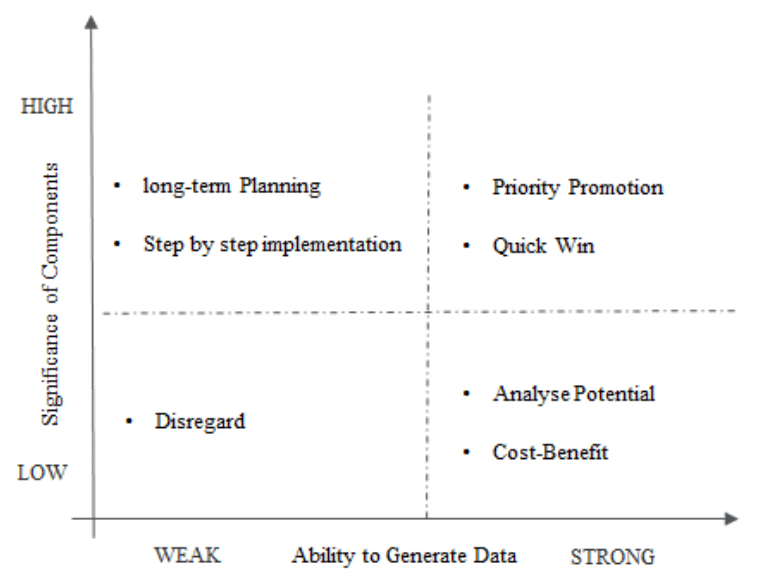

Figure 1 Priority Matrix of Predictive Maintenance Requirements

For areas with important assets and high data generation capacity, priority will be given to promote and achieve quick win results; For areas with important assets, but the ability to generate data is not perfect at present, long-term planning and step-by-step implementation shall be considered; For areas with nonimportant assets but strong data generation ability, we should analyse potential opportunities and promote selectively based on the principle of cost-benefit; For areas with non-important assets and weak data generation ability, do not spend too many resources on them for the time being. This strategy helps to ensure that the results are realistic before deploying resources.

\subsection{Build a Composite Team}

Practice has proved that effective predictive maintenance teams often need three types of core skilled talents, namely railway industry experts, data scientists and system design and development personnel. Combining data analysis with professional knowledge in the railway field to guide data scientists to build correct algorithms according to specific business needs, and then realize them through digital technology design and development, which will be the usual path to successfully implement predictive maintenance.

It is very important for railway experts and data analysis teams to develop algorithms for predictive maintenance solutions. Both data scientists with expertise in algorithm writing and railway experts proficient in industry insight should integrate their core competencies to develop intelligent solutions that can achieve predictive maintenance efforts with a certain accuracy.

\subsection{Create a Good Change Culture}

Railway personnel are used to the traditional maintenance methods. Therefore, it is a change for promoting predictive maintenance. On the one hand, railway managers need to strengthen their faith and vigorously promote predictive maintenance; On the other hand, predictive maintenance can not only predict a railway asset failure, but also predict several business scenarios. In these scenarios, appropriate prescriptive actions can be deployed to improve the performance indicators related to maintenance. This will include recommending the next maintenance activity, developing an inventory plan for parts replacement in a timely manner, and identifying systems that need to be upgraded due to continued poor performance to address short-term and long-term goals.

\section{ARCHITECTURE DESIGN OF PREDICTIVE MAINTENANCE PLATFORM BASED ON IOT AND AI TECHNOLOGY}

Predictive maintenance is developed based on Internet of things and AI technology. Although predictive maintenance has many different business application scenarios in railway, its common comprehensive platform architecture can be summarized as shown in Figure 2.

This platform should generally have the following capabilities to monitor and analyse railway asset health data, gain reasonable insights and provide them to relevant organizations or individuals for further action. 


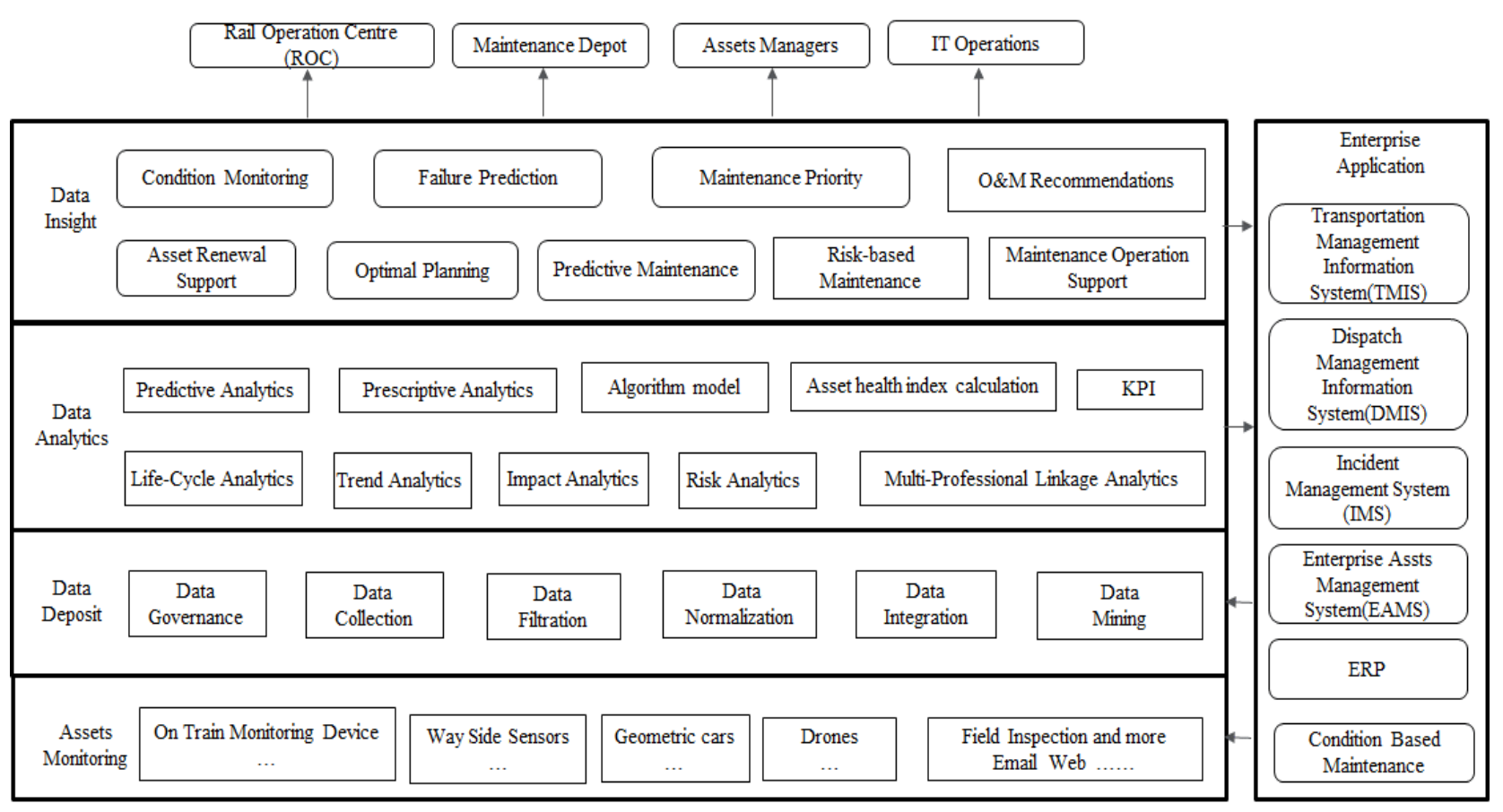

Figure 2 Architecture Design of Predictive Maintenance Platform

(1) Asset status monitoring: the platform shall carry various sensors to monitor the operation status of assets regularly / irregularly, and real-time monitoring is required at several key asset parts.

(2) Data capture and processing: the platform can capture asset data from various condition monitoring devices without being affected by data format, data source or sensor type. ${ }^{[6]}$ These raw data can be processed at the edge and stored in the database or file system according to the type.

(3) Data intelligence analysis: the platform should use the available sensor data along with other relevant data to develop meaningful insights using various analytics tools such as machine learning, algorithms for anomaly detection and real-time warning, predictive asset health assessment, stream analytics and so on. ${ }^{[7]}$ With dashboards based on historic data on asset condition and with predictive analysis of sensor data, the platform should enable business intelligence and drive superior decision-making.

(4) Data insight capability: Valuable insights can be obtained through data analysis, including failure prediction, maintenance sequencing, operation and maintenance suggestions, asset renewal e suggestions, plan optimization, predictive maintenance suggestions, decision-making operation, prediction opportunities, etc.

(5) Integration with enterprise applications: the platform should seamlessly integrate with enterprise asset management (EAM), data warehouse and other enterprise IT systems using a standard middleware platform to create a unified view and trigger events and workflows for subsequent activities.

\section{CONCLUSION}

China's railway development has been in the forefront of the world, but the application of predictive maintenance still lags behind developed countries. With the rapid development of domestic Internet of things and artificial intelligence technology in recent years, it is suggested that railway enterprises should make great efforts to invest resources in the development of predictive maintenance, think of the big, start with the small and promote rapidly. This literature has adequately demonstrated this aspect and recommends schema design approach as an enabler to implement predictive maintenance strategy in rail sector.

With regard to further work and future direction, it is prudent to approach the business scenario possibly with selecting several cases to verify the applicability of predictive maintenance framework scheme design. Finally, it is emphasized that applying AI techniques to data rich organization like Railways is expected to pay good dividends in terms of better predictive maintenance support towards maximizing capacity utilization, reliability of assets, safety etc. 


\section{REFERENCES}

[1] China State Railway Group Co., Ltd., Outline of Powerful Nation Railway Advance Planning in the New Era, 2020 ,

https://m.thepaper.cn/baijiahao_8707560

[2] Bergquist, B., Söderholm, P. Data Analysis for Condition-Based Railway Infrastructure Maintenance in: Quality and Reliability Engineering Interantional, 2014, DOI: 10.1002 /qre. 1634 .

[3] Stephan Menze, How Does Predictive Maintenance Succeed in: Electronic Engineering\& Product World, vol. 6, 2019, pp.22

[4] Min LIU, Ling LI, Feng YAN, Intelligent Predictive Maintenance, Chemical Industry Press, 2020
[5] Zhansheng WANG, Ling DING, Liqiang YANG, Ning ZHANG, Cluster Analysis on Urban Rail Transit Ticket Types in: Intelligent Computation Technology and Automation (ICICTA), 2010 International Conference on, vol.1, 2010, pp. 950953, DOI: 10.1109/ICICTA.2010.829

[6] Sammouri, Wissam, et al. Mining floating train data sequences for temporal association rules within a predictive maintenance framework. In: Advances in Data Mining. Applications and Theoretical Aspects. Springer Berlin Heidelberg, pp. 112-126, 2013.

[7] Jiang Feng WANG, Technical Basis and Application of Traffic Detection and Internet of Thing, Beijing JiaoTong University Press, 2020 